\title{
A Kallikrein 15 (KLK15) single nucleotide polymorphism located close to a novel exon shows evidence of association with poor ovarian cancer survival
}

Jyotsna Batra ${ }^{1,2}$, Christina M Nagle ${ }^{3}$, Tracy O’Mara ${ }^{1,2}$, Melanie Higgins ${ }^{1,2}$, Ying Dong ${ }^{1}$, Olivia L Tan ${ }^{1}$, Felicity Lose ${ }^{2}$, Lene Marie Skeie ${ }^{1}$, Srilakshmi Srinivasan ${ }^{1}$, Kelly L Bolton ${ }^{4,5}$, Honglin Song ${ }^{4}$, Susan J Ramus ${ }^{6}$, Simon A Gayther ${ }^{6}$, Paul DP Pharoah" ${ }^{4}$, Mary-Anne Kedda', Amanda B Spurdle ${ }^{2}$ and Judith A Clements ${ }^{1 *}$

\begin{abstract}
Background: KLK15 over-expression is reported to be a significant predictor of reduced progression-free survival and overall survival in ovarian cancer. Our aim was to analyse the KLK15 gene for putative functional single nucleotide polymorphisms (SNPs) and assess the association of these and KLK15 HapMap tag SNPs with ovarian cancer survival.

Results: In silico analysis was performed to identify KLK15 regulatory elements and to classify potentially functional SNPs in these regions. After SNP validation and identification by DNA sequencing of ovarian cancer cell lines and aggressive ovarian cancer patients, 9 SNPs were shortlisted and genotyped using the Sequenom iPLEX Mass Array platform in a cohort of Australian ovarian cancer patients $(N=319)$. In the Australian dataset we observed significantly worse survival for the KLK15 rs266851 SNP in a dominant model (Hazard Ratio (HR) 1.42, 95\% Cl 1.021.96). This association was observed in the same direction in two independent datasets, with a combined HR for the three studies of 1.16 (1.00-1.34). This SNP lies 15bp downstream of a novel exon and is predicted to be involved in mRNA splicing. The mutant allele is also predicted to abrogate an HSF-2 binding site.

Conclusions: We provide evidence of association for the SNP rs 266851 with ovarian cancer survival. Our results provide the impetus for downstream functional assays and additional independent validation studies to assess the role of KLK15 regulatory SNPs and KLK15 isoforms with alternative intracellular functional roles in ovarian cancer survival.
\end{abstract}

Keywords: Kallikrein 15 single nucleotide polymorphisms, ovarian cancer, splice variants

\section{Background}

Ovarian cancer is an aggressive disease with high metastatic potential and is frequently diagnosed at an advanced stage $[1,2]$. In vitro studies show that malignant cells synthesize and secrete proteolytic enzymes which disrupt basement and extracellular membranes to allow malignant cells to invade neighboring tissues and

\footnotetext{
* Correspondence: j.clements@qut.edu.au

'Australian Prostate Cancer Research centre-Queensland and Institute of Health and Biomedical Innovation, Queensland University of Technology, Kelvin Grove, Brisbane, Queensland, 4059, Australia

Full list of author information is available at the end of the article
}

metastasize [3]. Members of the Kallikrein-related (KLK) peptidase family are part of a proteolytic enzymatic cascade activated in aggressive forms of hormone-related cancers including ovarian cancer [4-6]. The KLKs are encoded by a 15 -member gene family clustered together in a region of approximately $320 \mathrm{~kb}$ on chromosome 19q13.4 [5-7]. KLK15 (encoding for KLK15, previously reported as $\mathrm{hK} 15$, or prostinogen) is the most recently cloned member of the human kallikrein gene family [8], sharing a high degree of structural similarity with KLK3 (PSA), and is positioned adjacent to the KLK3 gene [5,8]. KLK15 mRNA expression levels are up-regulated

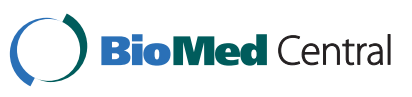

(c) 2011 Batra et al; licensee BioMed Central Ltd. This is an Open Access article distributed under the terms of the Creative Commons Attribution License (http://creativecommons.org/licenses/by/2.0), which permits unrestricted use, distribution, and reproduction in any medium, provided the original work is properly cited. 
in prostate cancer [9-11] and ovarian cancer [12]. In addition, KLK15 mRNA expression is reported to be a favorable diagnostic marker for breast cancer [13], and a significant predictor of reduced progression-free survival and overall survival after ovarian cancer diagnosis [12]. Several studies have been undertaken investigating the role of $K L K$ genetic variants in different cancers, aiming to better understand cancer biology and also to identify potential new targets for genetic testing with regard to cancer risk and prognosis [14-17]. Of note, we have recently identified a PSA promoter SNP to be associated with ovarian cancer survival (O'Mara, manuscript submitted), however, no studies have been undertaken to assess the role of $K L K 15$ genetic variation in ovarian cancer prognosis.

We used in silico approaches for data mining of the KLK15 gene for potential functional motifs and differential splicing to determine regions of the gene that could be functionally compromised by genetic variations. This was supplemented by identification of potentially functional common SNPs using database searches and resequencing of ovarian cancer cell lines and patients, to prioritize variants for ovarian cancer prognosis studies. We then assessed the association between ovarian cancer survival and 9 SNPs tagging 22 prioritized KLK15 SNPs in an Australian dataset, and undertook replication studies in two other ovarian cancer studies to validate our findings.

\section{Methods}

\section{Study Participants and Genotyping}

The initial phase of this study included 319 Australian women diagnosed with primary invasive epithelial ovarian cancer between 1985 and 1997. Over half of the women $(\mathrm{N}=207,65 \%)$ had participated in a large population-based case-control study of the etiology of ovarian cancer. Briefly, these women were ascertained through major gynecology-oncology treatment centres in the three most populous Australian states: Queensland, New SouthWales and Victoria. A central gynecologic histopathologist reviewed all pathology reports and sections of each tumor to confirm the diagnosis and histological subtype. The remaining women $(\mathrm{N}=112,35 \%)$ were ascertained as consecutive incident cases from the Royal Brisbane Hospital (RBH), Queensland, Australia. The patients in the study were included on the basis on availability of DNA and survival/follow up data. Information on diagnosis, disease stage (using the International Federation of Gynecologists and Obstetricians [FIGO] criteria), tumor histology and grade was abstracted retrospectively from the women's medical records and pathology reports or, for a subset of cases, from the RBH Gynecology Oncology database. Full details of the methods and the characteristics of the cases have been reported previously $[15,18]$. The women were followed for mortality using personal identifiers which were linked to the Australian National Death Index (NDI), State Cancer Registry records and the RBH Gynecology Oncology database. DNA was extracted as described in previous studies $[15,18]$ and genotyping of the KLK15 SNPs was performed using the Sequenom iPLEX MassArray platform (San Diego, CA, USA) according to manufacturer instructions. The first replication sample set included 1815 patients from the stage 1 of an ovarian cancer genome-wide association study (GWAS), all with confirmed invasive epithelial ovarian cancer from four different ovarian cancer case series in the United Kingdom (UK): United Kingdom Ovarian Population study (UKOPS), Study of Epidemiology and Risk Factors in Cancer Heredity (SEARCH), Royal Marsden Hospital (RMH) and United Kingdom Familial Ovarian Cancer Registry (UKFOCR), as described previously [19]. Genotyping of the replication sample set was performed as part of the first published genomewide association study (GWAS) of ovarian cancer [19].

The second replication dataset consisted of 413 women with invasive epithelial ovarian cancer from The Cancer Genome Atlas (TCGA) Pilot Project established by the National Cancer Institute and the National Human Genome Research Institute. Information about TCGA and the investigators and institutions that constitute the TCGA research network can be found at their website [20]. Genotyping and clinical data for ovarian cancer patients were downloaded via the TCGA data portal.

Progression-free survival, residual disease status and platinum sensitivity data were not available for the Australian or European sample sets. Informed consent was obtained from all the participating subjects. Human Ethics Committee approval was given for the recruitment and genotyping of all the individuals included in this study.

\section{Web based search for putative splice-variants}

Access information for the KLK15 gene and its protein product was downloaded from different databases as given in Additional file 1. Potential KLK15 alternative splice variants were identified through sequence alignment analysis of the splice variants and Expression Sequence Tags (ESTs) reported in publicly available databases including NCBI and Ensembl. Sequence alignments were performed using the clustal W platform. SignalP 3.0 Server and PSORT II Prediction default matrices were used to determine if each $K L K 15$ isoform contained a signal peptide. mRNA folding of all the KLK15 variants was analysed by in silico modeling using mfold version 2.3. Additional file 2 details the websites used for the in silico analysis. 


\section{In silico Promoter and SNP analysis}

The $11 \mathrm{~kb}$ region upstream of exon 1 on chromosome 19 (56020307 to $56037591 \mathrm{bp}$, NCBI build 36.3) along with the KLK15 coding sequence (Ensemble ID; ENSGG000000174562) was selected for putative promoter and SNP analysis using websites in Additional file 2. In brief, WWW Promoter Scan, PromoterInspector, CpG Islands, and Cister were used to predict and analyse putative promoter regions. Since hormonal steroids are known to be key regulators of $K L K$ expression in hormone dependent cancers [21,22], we assessed the promoter regions for putative estrogen and androgen regulatory elements. Dragon ERE finder (sensitivity of 0.83 ) and Cister (ERE matrix) were used to find putative estrogen response elements (EREs). Cister (ARE matrix) and JASPAR (threshold 75\%) were used to identify putative androgen response elements (AREs). SIFT and Polyphen were used to predict the functional significance of non-synonymous SNPs (nsSNPS). Webserver FastSNP was used for predicting the functional significance of the UTR and splice site SNPs. Analysis for putative microRNA (miRNA) sites was performed using miRBase Targets V4, Target scan, miRanda, PicTar and Patrocles.

\section{Cell culture, RT PCR and sequencing of KLK15 putative promoter region}

The normal ovarian cell line HOSE17.1 and serous epithelial ovarian carcinoma cell lines OVCA432, SKOV3, PEO1 were cultured as previously described [15]. RNA extraction and reverse transcription-PCR (RT-PCR) were performed as previously published [15]. Briefly, RNA was isolated using the RNAeasy kit (Qiagen), treated with DNaseI (Invitrogen), and $2 \mu \mathrm{g}$ RNA reverse-transcribed using Superscript III ${ }^{\mathrm{TM}}$ (Invitrogen). PCR was performed with primers (K15ExBFor, 5'-TTCAAGACCCCCAGATGGAGAAAAG-3' and K15Ex2Rev, 5'-CTTCCAGCAACTTGTCACCA-3') for 35 cycles with conditions of $94^{\circ} \mathrm{C}$ for $5 \mathrm{~min}$ followed by 35 cycles of $94^{\circ} \mathrm{C}, 64^{\circ} \mathrm{C}$ and $72^{\circ} \mathrm{C}$ for 1 min each, and a final extension at $72^{\circ} \mathrm{C}$ for $10 \mathrm{~min}$. PCR for $\beta 2$-microglobulin was used as an internal control. The PCR products were visualised by electrophoresis on $1.5 \%$ agarose gels stained with ethidium bromide. Genomic DNA was extracted using QIAamp DNA Mini Kit (Qiagen, Hilde, Germany). Four primer sets (Additional file 3) were designed using NETprimer http://www.premierbiosoft. com/netprimer/index.html to amplify segments of the putative $K L K 15$ promoter region with the greatest density of likely functional SNPs, based on results from the in silico analysis. All reactions $(20 \mathrm{uL})$ contained $10 \mathrm{ng}$ of genomic DNA, $0.25 \mathrm{uM}$ of each region specific primer (Proligo, Lismore, NSW, Australia), $0.2 \mathrm{mM}$ dNTP mix (Roche, Castle Hill, NSW, Australia), 0.03 mM Magnesium Chloride (Invitrogen, Victoria, Australia).
Denaturing agents DMSO and betaine (Sigma) were added to destabilize secondary structures in the template, and improve both the PCR and sequencing quality. PCR products were sequenced at the Australian Genome Research Facility (AGRF, Brisbane, Australia) using dideoxy dye chain-termination technology (Applied Biosystems). Sequences were aligned and analyzed using SeqMan ${ }^{\mathrm{TM} I I}$ (DNASTAR, WI, USA).

\section{Statistical Analysis}

Survival time was calculated from date of diagnosis to date of death (from ovarian cancer) or censored at 1 September, 2004 or death from another cause. The Kaplan-Meier technique was used to estimate crude overall survival probabilities, and adjusted hazard ratios (HR) and 95\% confidence intervals (CI) were obtained from Cox regression models adjusted for age group, FIGO stage, histologic subtype and grade. The TCGA dataset was adjusted for age, FIGO stage and histologic grade, but not subtype because all the samples were of the serous subtype. For the combined analysis, a weighted average of the log HR was calculated, taking into account random effects using the method of DerSimonian and Laird [23]. Linkage Disequilibrium (LD) maps were generated using Haploview 4.2 [24].

\section{Results}

\section{Analysis of $K L K 15$ transcripts}

All of the published KLK15 mRNA variants [25] were collated from GenBank (Figure 1A). Six isoforms were observed. The classical form, isoform 4 (NM_017509), has five exons and encodes a protein of 256 amino acids (aa). However, this was recently extended to include an additional 5' untranslated exon (exon A) discovered from an EST clone (CF139951). Another 3 known isoforms (1-3) encode 3 different length proteins of 122aa, 161 aa and 171 aa respectively. Isoform 5 (AY373373) and isoform 6 (AY373374) both encode proteins of 162aa, since they share the same stop codon at the beginning of intron 3; the additional loss of exon 4 for isoform 6 does not affect protein length (Figure 1A). Isoform 5 was found to form the most stable secondary structure based on $\Delta G$ values predicted by mfold, while isoform 3 was the least stable (data not shown).

We also identified an additional KLK15 splice variant from the three new EST clones (GenBank accession No. BP428687, BP431267, BP431386). This new isoform has a novel exon between exon $A$ and exon 1, that we have named exon B. Exon B is located 1575 bp upstream from exon 1, however all three EST clones show that exon $\mathrm{B}$ is spliced onto exon 2, skipping exon 1 (Figure 1A). The longest clone encodes a protein that is 106 aa long, shorter than that observed for the other isoforms; however it does not contain a stop codon and therefore 




is likely to be an incomplete sequence (Figure 1A). Using SignalP 3.0 Server and PSORT II, the exon B variant was not predicted to contain a possible cleavage site for an $\mathrm{N}$-terminal signal peptide, thus is most likely to be an intracellular protein. In contrast, the other isoforms all are predicted to have signal peptides and a predominantly cytoplasmic and extracellular localization as expected for secreted proteins. The exon $\mathrm{B}$ variant was confirmed by RT-PCR in serous ovarian epithelial cancer cell lines PEO1 and OVCA432 and also in the prostate cancer LNCaP cell lines (Figure 1B).

\section{In silico promoter analysis to prioritize regions for sequencing and SNP modeling}

Cister and JASPAR together identified 30 putative Androgen Response Elements (AREs) (15 upstream of exon A, 1 in exon $\mathrm{A}, 8$ between exon $\mathrm{A}$ and $\mathrm{B}, 2$ in exon $\mathrm{B}$, and 4 between exon $B$ and 1). Only 2 AREs (Figure 2A) were predicted by both tools (1 upstream of exon A and the other between exon B and exon 1). Dragon ERE finder and Cister predicted 30 putative Estrogen Response
Elements (EREs) upstream of exon 1 (17 upstream exon A, 9 between exon A and B, and 4 between exon $B$ and 1 ). Four EREs (Figure 2A) predicted by Dragon ERE finder overlapped an ERE predicted by Cister. Cister identified two clusters of cis-elements (Figure 2A), both upstream of exon A. The first cluster starts $4232 \mathrm{bp}$ upstream of exon A, spans $467 \mathrm{bp}$ and contains eight putative cis-elements. The second cluster starts 2108 bp upstream of exon A, spans $1892 \mathrm{bp}$ and contains 10 putative cis-elements. Two putative promoter regions on the forward strand were detected using WWW Promoter Scan The first putative promoter is $189 \mathrm{bp}$ downstream of exon A and spans 250 $\mathrm{bp}$, and the second is $852 \mathrm{bp}$ downstream of exon $\mathrm{A}$ and spans $252 \mathrm{bp}$. PromoterInspector detected one putative promoter region which is 992 bp downstream of exon A and spans $463 \mathrm{bp}$. CpGPlot showed three putative CpG islands with a percent $\mathrm{C}+\mathrm{G}$ of above $50 \%$, observed/ expected ratio of above 0.60 and a length of over $100 \mathrm{bp}$ : the first is $4519 \mathrm{bp}$ upstream of exon A and spans $115 \mathrm{bp}$; the second is $538 \mathrm{bp}$ downstream of exon A and spans $106 \mathrm{bp}$; the third is $778 \mathrm{bp}$ downstream of exon $\mathrm{A}$ and 


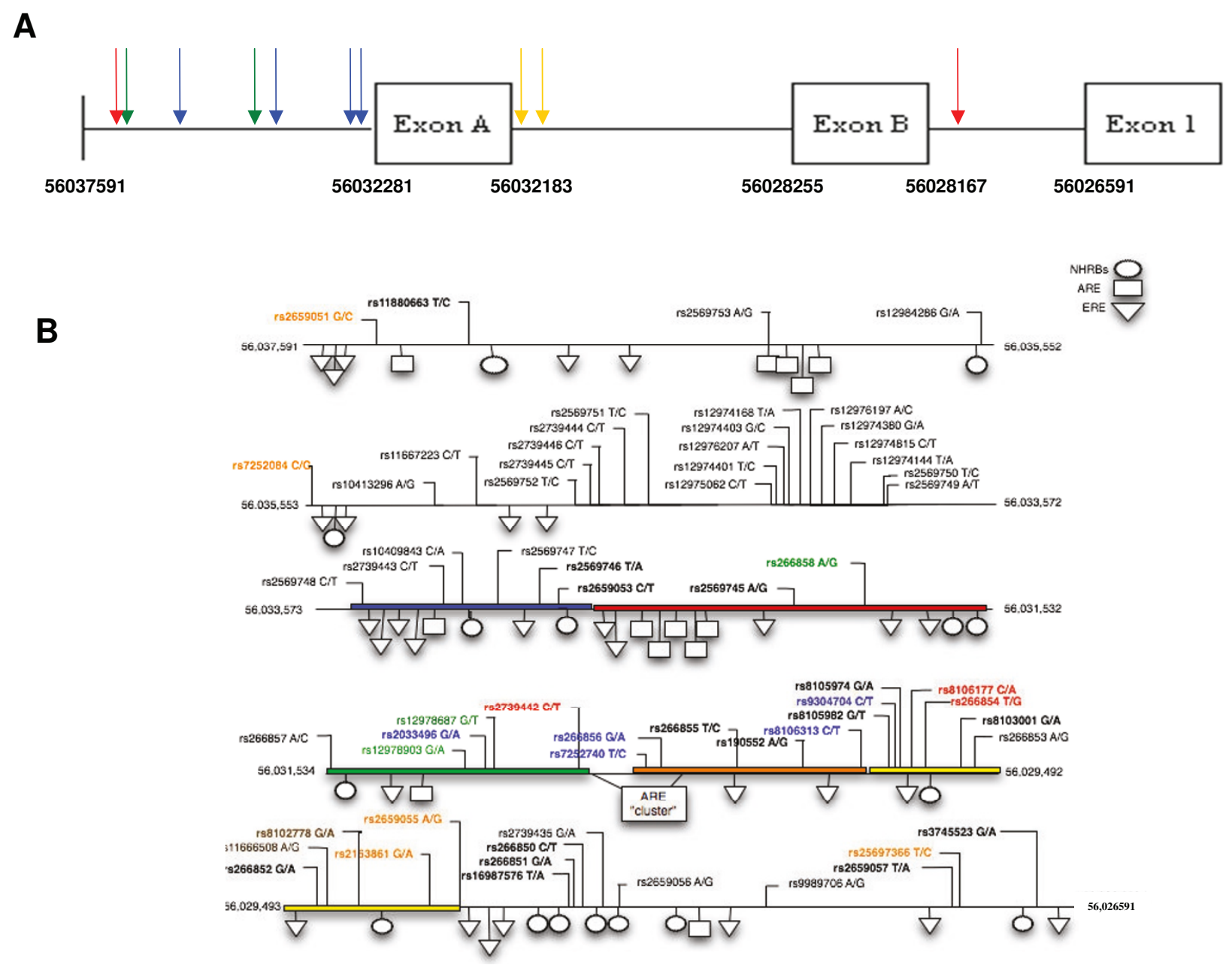

Figure 2 The putative KLK15 promoter region: (A) The 11 kb region upstream of exon 1 on chromosome 19 (56020307 to 56037591 bp, NCBI build 36.3) was downloaded for in silico analysis (A) schematic of the $5^{\prime}$ region of the KLK15 gene with significant motifs noted: 2 putative Androgen Response Elements (AREs) were found by both JASPAR and Cister programs (red arrows); 4 putative Estrogen Response Elements (ERES) were found by both ERE finder and Cister programs (blue arrows); 2 putative cis-element clusters which indicate putative promoter regions found by Cister (green arrows); 2 putative promoter regions that overlap with CpG islands found by WWWPromoter Scan, Promoter Inspector and CpG Island Finder (yellow arrows) (B) AREs (boxes), EREs (triangle) and Nuclear Hormone Receptor Binding Sites (NHRBs) (circles) as predicted by individual softwares. The ARE "cluster" consists of 16 AREs. The colored bars (blue, red, green, orange, yellow) in the sequence indicate the 5 regions that were chosen for sequencing genomic DNA. The single nucleotide polymorphism (SNP)-modeling results are shown by color coding the functional SNPs validated in bold. Orange and red text coloring indicate a gain or loss of ERE motifs respectively, while green and blue indicate ARE gain or loss. The two brown SNPs indicate a loss of one ERE and a loss of two AREs. An additional four validated SNPs viz rs2659052, rs1 1880663, rs2659051, rs2659055 have been added to NCBI databases (not modeled here) from the time of our analysis.

spans 564 bp. The last two putative CpG islands overlap putative promoters found by WWW Promoter Scan and PromoterInspector (Figure 2A).

\section{SNP information and prediction of functional effects Gene}

A total of 76 (27 validated) SNPs in the exonic-intronic region of KLK15 were retrieved from the NCBI dbSNP database, comprising 10 exonic SNPs (4 non-synonymous, 1 synonymous and 5 in 3' UTR) (Additional file 4) and 66 intronic SNPs (data not shown). Although predicted to be damaging by FastSNP, SIFT and PolyPhen predicted that the validated non-synonymous (ns) exonic SNPs were unlikely to have any deleterious effect on protein structure (Additional file 4). FastSNP server predicted that the synonymous (s) SNP and the 3' UTR SNPs were not damaging with a risk score of 0-1 (Additional file 4). The rs3212852 SNP present at the 3' end of exon 3 was predicted to have a damaging effect with a medium-high risk score of 3-4. No damaging scores were observed for the 
additional 5 intronic SNPs located within $30 \mathrm{bp}$ of exonintron boundaries. We also predicted miRNA binding sites using three different software programs; Target Scan, miRanda and Patrocles. A maximum of 32 miRNA binding sites scattered throughout the gene were predicted by miRanda (data not shown). Interestingly, the 3'UTR SNP rs3212810 lies in the target sequence of mir-498, which has been reported to be dysregulated in prostate cancers [26]. Also, rs3212853 was predicted to be a potential polymorphic miRNA target site (using Patrocles target database), where the presence of the mutant allele creates a binding site for miR-193b (Additional file 4), shown to be involved in tumor progression and invasion in human breast cancer [27] and a tumor suppressor in prostate cancer [28].

\section{Promoter}

As gene expression may be influenced by promoter polymorphisms, we included analysis of SNPs in the 11 $\mathrm{kb}$ upstream region from exon 1 and found 62 (out of the total 101) validated SNPs. In silico analysis predicted that 8 SNPs created a new putative ERE/ARE site while 10 other SNPs were predicted to destroy one or more putative ERE/ARE sites using two different software programs; JASPAR and Cister for the ARE and Cistor and Dragon ERE finder for the ERE (Figure 2B). Further, it was observed that 22 SNPs created one or more new transcription factor binding sites (TFBS) while 25 SNPs completely lost one or more TFBS as predicted by Genomatrix ElDorado program (data not shown).

\section{Promoter sequencing of cancer cell lines and aggressive cancer patients}

To confirm the polymorphic status of reported SNPs and identify novel SNPs with potential prognostic significance, we sequenced genomic DNA within the KLK15 promoter (Figure 2B) of four ovarian (normal -HOSE17.1; cancerOVCA432, SKOV3 and PEO1) cell lines and the germline DNA of 30 ovarian cancer patients with aggressive disease. Sequencing analysis of the cell lines validated 15 SNPs, including 6 non-validated SNPs in the NCBI database (Additional file 5). Sequencing of genomic DNA from aggressive ovarian cancer patients validated additional 5 SNPs (Additional file 5), and identified two novel SNPs that were not predicted to be functionally relevant. Seven SNPs from the NCBI database were found to be non-polymorphic in our sequencing cohort. The putative functional role of the promoter SNPs is provided in Additional file 5.

\section{LD mapping and SNPs selection for survival studies}

SNPs chosen for genotyping in this study were (i) identified as tagging SNPs using HapMap version 22 (April 07 ), using a minor allele frequency $>0.05$ and pairwise linkage disequilibrium threshold of $\mathrm{r}^{2}>0.8$ (rs2659058, rs3212810, rs3745522, rs2659056, rs266851, rs2163861, rs266856), or (ii) chosen due to the above reported in silico prediction of functional effect on KLK15 expression (rs3212853, rs3212852, rs16987576, rs2659055, rs266853, rs266854, rs190552, rs266855, rs2739442, rs2033496, rs12978902, rs2659053, rs2569746, rs35711205, rs2569747) (Table 1). As the frequency data for many of these SNPs were not available, we genotyped all 22 SNPs in controls and generated the LD map using Haploview 4.2 (Batra et al, unpublished data; Additional File 6). All SNPs except rs3745522 were found to follow HardyWeinberg equilibrium ( $\mathrm{p}<0.01)$. SNP rs12978902 was non-polymorphic, while rs3212853, rs3212852, rs16987576 rs266853 and rs266854 were found to have minor allele frequencies $<0.05$ (Table 1 ), so were not pursued further for the survival analysis. SNPs in high LD with other SNPs $\left(r^{2}>0.9\right)$ were also not pursued further. Priority was given to putative functional SNPs, with a total of 12 SNPs shortlisted for further analysis (Table 1). Three of these SNPs (rs2659056, rs266855 and rs35711205) could not be genotyped in ovarian cancer samples because of assay incompatibilities.

\section{Association with ovarian cancer survival}

Among the 319 Australian women with ovarian cancer, 188 (59\%) died from the disease during the follow-up period, with a 5 -year survival proportion of $45 \%$. Selected clinical and pathologic characteristics of the Australian women and the women in the two independent datasets, the UK GWAS and TCGA, are shown in Table 2. Among the Australian women a little over three quarters were older than $\geq 50$ years at diagnosis (77\%), and many presented with late stage disease $(71 \%)$, tumors of high grade (54\%) and serous histological subtype (64\%). In the UK GWAS dataset most women were $\geq 50$ years at diagnosis $(78 \%)$, just over half the group had early stage disease (56\%) and serous histological subtype (54\%). The TCGA dataset comprised women who were mostly older than $\geq 50$ years at diagnosis $(79 \%)$, and the majority had late stage (95\%), high grade (88\%) disease and all were of serous histologic subtype.

SNP rs266851 showed statistically significant evidence for an association with worse survival (Table 3) in the Australian dataset. Increased risk was observed for the heterozygote genotype CT (HR 1.43, 95\% CI 1.02-2.00), and also for the rare (2\% of sample) homozygote genotype (HR 1.26 , 95\% CI 0.49-3.24); p trend $=0.01$. Overall association under a dominant model was HR 1.42 with $95 \% \mathrm{CI}=1.02$ 1.96 (Table 3). This SNP was not found to be associated with ovarian cancer stage $(\mathrm{p}=0.41)$ or grade $(\mathrm{p}=0.39)$ (Table 2). None of the other SNPs investigated were associated with ovarian cancer survival in Australian dataset.

In an attempt to replicate our results, we analyzed genotype data obtained from the UK GWAS and the 
Table 1 SNP selection for the survival studies

\begin{tabular}{|c|c|c|c|c|c|c|c|}
\hline SNP no & Name & Rationale & Position & ObsHET & PredHET & HWpval & MAF \\
\hline 1 & rs2659058 & Tagged SNP from HapMap & 56017918 & 0.421 & 0.443 & 0.0869 & 0.331 \\
\hline 2 & rs3212810 & Tagged SNP from HapMap and predicted to fall in miRNA binding site & 56020548 & 0.364 & 0.369 & 0.6072 & 0.244 \\
\hline 3 & rs3212853 & Ploymorphic miRNA target site & 56020606 & 0.044 & 0.041 & 0.2863 & 0.037 \\
\hline 4 & rs3212852 & Exon-Intron boundary & 56021941 & 0.038 & 0.038 & 1 & 0.019 \\
\hline 5 & rs3745522 & Tagged SNP from HapMap & 56022744 & 0.349 & 0.383 & 0.0013 & 0.259 \\
\hline 6 & rs2659056 & Tagged SNP from HapMap & 56027755 & 0.383 & 0.382 & 1 & 0.257 \\
\hline 7 & rs266851 & Tagged SNP from HapMap & 56028151 & 0.297 & 0.306 & 0.2742 & 0.189 \\
\hline 8 & rs16987576 & 2 bp $3^{\prime}$ of exon B, Imp for splicing & 56028165 & 0.003 & 0.003 & 1 & 0.001 \\
\hline 9 & rs2659055 & Predicted in silico to affect HRE & 56029044 & 0.485 & 0.5 & 0.3033 & 0.49 \\
\hline 10 & rs2163861 & Tagged SNP from HapMap & 56029204 & 0.485 & 0.5 & 0.2841 & 0.489 \\
\hline 11 & rs266853 & Predicted in silico to affect TFBS and HRE & 56029520 & 0.001 & 0.001 & 1 & 0.001 \\
\hline 12 & rs266854 & Predicted in silico to affect HRE & 56029630 & 0.004 & 0.004 & 1 & 0.002 \\
\hline 13 & rs190552 & Predicted in silico to affect TFBS and HRE & 56030005 & 0.333 & 0.346 & 0.1823 & 0.222 \\
\hline 14 & rs266855 & Predicted in silico to affect HRE & 56030196 & 0.396 & 0.413 & 0.1259 & 0.292 \\
\hline 15 & rs266856 & Tagged SNP from HapMap & 56030318 & 0.333 & 0.349 & 0.1131 & 0.225 \\
\hline 16 & rs2739442 & Predicted in silico to affect TFBS and HRE & 56030985 & 0.479 & 0.493 & 0.2975 & 0.442 \\
\hline 17 & rs2033496 & Predicted in silico to affect TFBS and HRE & 56031019 & 0.49 & 0.493 & 0.8309 & 0.442 \\
\hline 18 & rs12978902 & Predicted in silico to affect HRE & 56031056 & 0 & 0 & 1 & 0 \\
\hline 19 & rs2659053 & Predicted in silico to affect TFBS and HRE & 56032606 & 0.449 & 0.472 & 0.0722 & 0.382 \\
\hline 20 & rs2569746 & Predicted in silico to affect TFBS and HRE & 56032727 & 0.462 & 0.487 & 0.0638 & 0.42 \\
\hline 21 & rs35711205 & Novel SNP (reported later in NCBI) & 56032818 & 0.296 & 0.309 & 0.1212 & 0.191 \\
\hline 22 & rs2569747 & Predicted in silico to affect TFBS and HRE & 56032875 & 0.459 & 0.487 & 0.0346 & 0.419 \\
\hline
\end{tabular}

ObsHet: Observed heterozygous alleles; PredHET: Predicted heterozygous alleles; HWpval: Hardy-Weinberg equilibrium p value; MAF: Minor allele frequency; HRE: Hormone Response Element; TFBS: Transcription Factor Binding Site.

SNPs in the KLK15 gene derived from the HapMap database and those by in silico prediction methods were genotyped in control individuals, and the minor allele frequency (MAF) and HWE were calculated using Haploview 4.2. SNPs in bold were shortlisted for genotyping and survival analysis on the basis on LD calculations.

TCGA datasets of ovarian cancer survival. We observed a similar direction of association in both the datasets for the dominant model (HR 1.07, 95\% CI 0.94 -1.24 in UK GWAS data and HR 1.20, 95\% CI 0.90-1.61 in TCGA data) (Table 4). Combining the results of these two studies (UK GWAS and TCGA data) with our data gave a summary HR of 1.16 (95\% CI 1.0-1.35) for the SNP rs266851 under a dominant model (Table 4).

\section{Putative functional elements associated with SNP rs266851}

The rs266851 SNP is predicted to have a FastSNP score $=0$ in our initial analysis. As the FastSNP algorithm is weighted by the SNP position with respect to gene structure, and our data shows that rs266851 SNP is located 15 bp 3' of a novel exon B, we used ESEfinder [29] to predict the putative role of rs266851 SNP in mRNA splicing. ESEfinder prediction suggests loss of various SRp binding sites in the presence of $\mathrm{T}$ allele (Additional file 7), thus this SNP could be involved in alternative mRNA splicing.

Our preliminary unpublished data (Lai et al., personal communication) suggest that exon B could be an alternative transcription start site (data not shown). Thus, using the TFbind webtool [30], we predicted that the $\mathrm{C}$ to $\mathrm{T}$ change may abrogate the binding site for Heat Shock Factor 2 (HSF2), which activates expression of Heat Shock Proteins (HSP), which have been shown to play a vital role in tumorigenesis [31,32].

\section{Discussion}

With the completion of the human genome project, an enormous amount of data has been generated and been made available to the research community through various database repositories. At the same time many bioinformatic/in silico tools have been made available to extract data and help conceptualize future studies. To our knowledge, this is the first study to use a comprehensive in silico approach for genetic data mining and regulatory region identification to direct SNP prioritization studies of the KLK15 gene and to analyze the association of KLK15 polymorphisms with ovarian cancer survival.

In our efforts to delineate the functional significance of the exonic SNPs, we used the Polyphen and SIFT algorithms which showed minimal associations of SNPs with likely functional amino-acid replacements. We thus proposed that SNPs in regulatory regions can act in cis 
Table 2 Summary of clinical and pathological factors in the Australian, UK GWAS and TCGA ovarian cancer datasets

\begin{tabular}{|c|c|c|c|}
\hline & $\begin{array}{l}\begin{array}{l}\text { Australian } \\
\text { dataset }\end{array} \\
\mathrm{n}^{*}(\%)\end{array}$ & $\begin{array}{l}\text { UK GWAS } \\
\text { dataset } \\
\mathrm{n}^{*}(\%)\end{array}$ & $\begin{array}{l}\text { TCGA } \\
\text { dataset } \\
\mathrm{n}^{*}(\%)\end{array}$ \\
\hline \multicolumn{4}{|l|}{ Age group } \\
\hline$<40$ & $21(7)$ & $97(5)$ & $7(2)$ \\
\hline $40-49$ & $51(16)$ & $316(17)$ & $74(19)$ \\
\hline $50-59$ & $85(26)$ & $653(36)$ & $123(31)$ \\
\hline $60-69$ & $99(31)$ & $594(33)$ & $97(24)$ \\
\hline $70+$ & $63(20)$ & $155(9)$ & $96(24)$ \\
\hline \multicolumn{4}{|l|}{ FIGO stage } \\
\hline I & $54(18)$ & $586(44)$ & $8(2)$ \\
\hline$\|$ & $35(11)$ & $162(12)$ & $12(3)$ \\
\hline III & $192(63)$ & $507(38)$ & $312(79)$ \\
\hline IV & $26(8)$ & $80(6)$ & $65(16)$ \\
\hline \multicolumn{4}{|l|}{ Histological grade } \\
\hline well differentiated & $41(14)$ & $239(18)$ & $2(1)$ \\
\hline $\begin{array}{l}\text { moderately } \\
\text { differentiated }\end{array}$ & $94(32)$ & $418(32)$ & $44(11)$ \\
\hline poor/undifferentiated & $156(54)$ & $643(50)$ & $344(88)$ \\
\hline \multicolumn{4}{|l|}{ Histological subtype } \\
\hline serous & $199(64)$ & $867(54)$ & $397(100)$ \\
\hline endometrioid & $35(11)$ & $200(13)$ & \\
\hline mucinuous & $23(8)$ & $320(20)$ & \\
\hline clear cell & $21(7)$ & $173(11)$ & \\
\hline other & $32(10)$ & $40(2)$ & \\
\hline
\end{tabular}

*Numbers may not sum to total due to missing data.

or trans, by leading to gain or deletion of a TFBS. In order to identify the $K L K 15$ promoter-regulatory region, multiple alignments of all the KLK15 splice variants and ESTs available in databases identified a novel isoform with a new exon (termed exon B), upstream of exon 1. This isoform utilizes exon B instead of exon 1 and is predicted to have a different function by encoding a protein lacking a signal peptide. Alternative $K L K$ transcripts may possess both physiological and prognostic significance and some are emerging candidate biomarkers $[25,33,34]$. For example, the PSA-RP2 [35] and KLK15 isoform $3[11,36]$ are upregulated in prostate cancer compared with benign prostatic hyperplasia tissues suggesting that differential mRNA splicing may be an important regulatory event in carcinogenesis. Thus, the novel exon $\mathrm{B}$ variant identified by this study needs to be explored further to assess its biological function and prognostic significance, and such studies are underway in our laboratory.

In order to assess the role of common genetic variation in altered regulation of $K L K 15$, and with the knowledge that steroid hormones have been implicated in the etiology and/or progression of epithelial ovarian cancer [3], we analyzed the in silico recognized promoter regions for
Table 3 Association between KLK15 Single Nucleotide Polymorphisms and ovarian cancer survival in Australian dataset

\begin{tabular}{|c|c|c|c|c|}
\hline KLK15 & $n$ & $\begin{array}{c}n \\
\text { censored }\end{array}$ & $\begin{array}{c}\text { Adjusted* HR }(95 \% \\
\text { CI) }\end{array}$ & $\begin{array}{l}p \text { value } \\
\text { (trend) }\end{array}$ \\
\hline \multicolumn{5}{|c|}{$r s 2659058$} \\
\hline$\pi$ & 123 & 74 & 1.0 & \\
\hline $\mathrm{CT}$ & 138 & 88 & $1.13(0.82-1.55)$ & 0.75 \\
\hline CC & 50 & 26 & $1.20(0.75-1.91)$ & \\
\hline \multicolumn{5}{|c|}{$r s 3212810$} \\
\hline CC & 207 & 122 & 1.0 & \\
\hline $\mathrm{TC}$ & 87 & 51 & $1.01(0.72-1.41)$ & 0.29 \\
\hline$\pi$ & 18 & 14 & $1.46(0.82-2.62)$ & \\
\hline \multicolumn{5}{|c|}{ rs3745522 } \\
\hline GG & 187 & 106 & 1.0 & \\
\hline GT & 81 & 48 & $0.97(0.68-1.39)$ & 0.15 \\
\hline$\pi$ & 18 & 13 & $1.73(0.93-3.24)$ & \\
\hline \multicolumn{5}{|c|}{$r s 266851$} \\
\hline CC & 222 & 128 & 1.0 & \\
\hline$C T$ & 89 & 55 & $1.43(1.02-2.00)$ & \\
\hline$\pi$ & 7 & 5 & $1.26(0.49-3.24)$ & 0.01 \\
\hline $\mathrm{CT} / \mathrm{TT}$ & 96 & 60 & $1.42(1.02-1.96)$ & \\
\hline \multicolumn{5}{|c|}{$r s 2659055$} \\
\hline$\pi$ & 84 & 52 & 1.0 & 0.23 \\
\hline $\mathrm{TC}$ & 144 & 87 & $0.86(0.60-1.23)$ & \\
\hline CC & 67 & 36 & $0.74(0.47-1.17)$ & \\
\hline \multicolumn{5}{|c|}{ rs190552 } \\
\hline$\pi$ & 178 & 109 & 1.0 & 0.79 \\
\hline$C T$ & 114 & 65 & $1.04(0.76-1.42)$ & \\
\hline CC & 19 & 13 & $1.21(0.67-2.17)$ & \\
\hline \multicolumn{5}{|c|}{$r s 2739442$} \\
\hline GG & 92 & 55 & 1.0 & 0.99 \\
\hline GA & 133 & 76 & $1.09(0.75-1.58)$ & \\
\hline$A A$ & 89 & 54 & $1.08(0.73-1.62)$ & \\
\hline \multicolumn{5}{|c|}{$r s 2659053$} \\
\hline GG & 129 & 79 & 1.0 & 0.2 \\
\hline GA & 118 & 73 & $0.93(0.66-1.29)$ & \\
\hline AA & 63 & 34 & $0.95(0.63-1.44)$ & \\
\hline \multicolumn{5}{|c|}{ rs2569746 } \\
\hline AA & 111 & 65 & 1.0 & 0.43 \\
\hline TA & 119 & 74 & $0.90(0.64-1.27)$ & \\
\hline$\pi$ & 57 & 36 & $1.12(0.74-1.69)$ & \\
\hline
\end{tabular}

${ }^{*}$ Adjusted for age, FIGO stage, histological subtype, and grade.

putative AREs and EREs. Though the results varied using different databases, overlapping predictions suggested the gain/loss of AREs and EREs in this region. We thus prioritized and confirmed the polymorphic status of the SNPs in this region by sequencing genomic DNA from four ovarian cell lines and 30 ovarian cancer patients. Of these putative functional and HapMap tag SNPs selected for genotyping, rs266851 was found to be associated with poor overall survival in ovarian cancer in the Australian cohort, The results were not found to be statistically 
Table 4 Results of ovarian cancer survival analysis for the KLK15 rs266851 SNP in Australian, UK GWAS data, TCGA data and the combined datasets

\begin{tabular}{llll}
\hline KLK15 rs266851 & $\mathbf{n}$ & $\mathbf{n}$ censored & Adjusted $^{*}$ HR $(\mathbf{9 5 \%} \mathrm{Cl}) *$ \\
\hline Australian & & & \\
CC & 222 & 128 & 1.0 \\
CT/TT & 96 & 60 & $1.42(1.02-1.96)$
\end{tabular}

$\begin{array}{llll}\text { UK GWAS** } & & \\ \text { CC } & 1139 & 390 & 1.0 \\ \text { СТ/TT } & 676 & 221 & 1.07(0.94-1.24)\end{array}$

$T C G A^{* * *}$

$\begin{array}{llll}\mathrm{CC} & 280 & 135 & 1.0 \\ \mathrm{CT} / \mathrm{TT} & 133 & 76 & 1.20(0.90-1.61)\end{array}$

Combined Analysis

\begin{tabular}{llll}
$\mathrm{CC}$ & 1641 & 653 & 1.0 \\
$\mathrm{CT} / \mathrm{TT}$ & 905 & 357 & $1.16(1.00-1.34)$ \\
\hline
\end{tabular}

*Adjusted for age, FIGO stage, histological subtype, and grade.

**The UK GWAS dataset was adjusted for age, FIGO stage, histologic subtype, grade and study site.

***The TCGA dataset was adjusted for age, FIGO stage and histologic grade, but not subtype as all the samples were of the serous subtype.

significant in the TCGA and UK GWAS cohorts but were similar in magnitude and direction to that of Australian dataset. This is what might be expected for "winner's curse" phenomenon, where the estimated effect of a marker allele from the initial study reporting the association is often exaggerated relative to the estimated effect in follow-up studies. The difference in magnitude of risk estimates may also be partially explained by different case ascertainment criteria. In particular, follow-up of the large majority of the UK GWAS dataset was initiated at least 6 months after diagnosis through the cancer registry, biasing against recruitment of ovarian cancer cases with short-term survival after diagnosis, and resulting in a dataset that included more women with early FIGO stage disease (56\% vs. $21 \%$ FIGO stage I and II) and fewer women diagnosed with serous ovarian cancer ( $47 \%$ vs. $66 \%$ ). Consequently the 5 -year survival rate was much higher in the UK GWAS dataset (77\% compared to $45 \%$ for the Australian dataset), and it is interesting to note that the UK dataset showed the risk estimate of lowest magnitude ( $H R=1.07)$. In contrast, the TCGA dataset with similar characteristics to the Australian dataset showed a risk estimate of greater magnitude $(H R=1.20)$, although still not as great as the initial risk estimate observed for the Australian dataset (HR 1.42). The overall pooled HR of 1.16 observed for the dominant model in our combined analysis is consistent with an association between rs266851 SNP and ovarian cancer survival in Australian dataset, and it would be important to confirm these findings in a larger sample set of incident cases.
Interestingly, the rs266851 SNP was found to be associated with an increased risk of breast cancer in the Cancer Genetics Markers of Susceptibility (CGEMS) project Breast Cancer GWAS [37] with a trend $\mathrm{p}$ value $=0.008$; heterozygote risk $=1.22(1.05-1.41)$; homozygote risk = 1.49 (1.11-2.00) (cases $\mathrm{N}=1145$, controls $\mathrm{N}=1142$ ).

So far no other SNP has been found to be in complete LD with rs266851 from HapMap and next generation sequencing data. Using in silico analysis, we predicted that rs266851 could potentially be involved in differential mRNA splicing as the mutant allele was found to abrogate the binding site for the SRp55 and SF2/ASF splicing factors. There are several reports of elevated SR protein family expression associated with ovarian cancer [38] and SF2/ASF, specifically, has been described as a proto-oncogene [39]. We also propose that rs266851 might be involved in regulation of KLK15 transcription by altering the HSF-2 binding site. Recently an HSF-2 binding site has been detected in the KLK5 and KLK7 promoter-regulatory regions and their expression has been found to correlate with HSF-2 expression in microarray analysis of breast malignancies [40]. Nevertheless, more experimental evidence is required to understand the effects of this variant on KLK15 expression and/or splicing.

\section{Conclusions}

This study supports a role of the KLK15 gene in ovarian cancer by providing suggestive evidence for an association of the rs266851 SNP with ovarian cancer survival. The location of this SNP adjacent to a novel exon B and in putative HSF2 and SRp binding sites should provide impetus for downstream functional assays and additional independent validation studies to assess the role of KLK15 regulatory SNPs and KLK15 isoforms with alternative intracellular functional roles in ovarian cancer survival. Our study also has applicability to studies investigating the role of $K L K 15$ genetic variation in other hormone-related cancers, namely prostate, endometrial and breast cancer.

\section{Additional material}

Additional file 1: Access information for KLK15. Information for KLK15 gene and its protein product was obtained from different databases and is outlined in the additional file1.

Additional file 2: In silico promoter and SNP analysis. Additional file 2 details the different websites and software used to scan the promoter region of KLK15 and to predict the functional significance of single nucleotide polymorphisms in and around the gene.

Additional file 3: Primer sequences. Additional file 3 details the primer sequence used for sequencing the KLK15 promoter region

Additional file 4: KLK15 intragenic SNPs and the results of in silico analysis. Additional file 4 details the SNPS in KLK15 exon and exon Intron boundary and the results of in silico analysis on these SNPS. 
Additional file 5: Validated KLK15 promoter SNPs. SNPS validated by KLK15 promoter sequencing of genomic DNA from ovarian cell lines and aggressive patients and their predictive functional role.

\section{Additional file 6: Linkage Disequilibrium map generated by}

Haploview 4.2. Frequency data was generated for the control individuals and the LD map was plotted. SNPs not in bold were found to have frequencies $<0.05$.

Additional file 7: Prediction of functional role of the rs266851 SNP ESE Finder Matrices analysis for SRp40, SC35, SF2/ASF and SRp55 splicing proteins.

\section{Acknowledgements and Funding}

We thank the patients and control subjects who participated so willingly in our studies. For the Australian sample sets, the authors are very grateful to staff at the Australian Red Cross Blood Services for their assistance with the collection of risk factor information and blood samples of healthy donor controls. We thank Georgia Chenevix-Trench for access to DNA samples and associated data for the ovarian cancer and SNP association studies. We thank the SEARCH team and the Eastern Cancer Registration and Information Centre. The results published here are in part based upon data generated by The Cancer Genome Atlas (TCGA) Pilot Project established by the $\mathrm{NCl}$ and NHGRI. Information about TCGA and the investigators and institutions who constitute the TCGA research network can be found at http://cancergenome.nih.gov.

ABS and JAC are NHMRC Senior and Principal Research Fellows respectively. CMN is supported by an NHMRC Career Development Award. JB and OLT are supported by Institute of Health and Biomedical Innovation Postdoctoral Fellowships. TOM is supported by an Australian Postgraduate Award complemented with an $\mathrm{HBI}$ and QLD Government Smart State Postgraduate Awards. SEARCH is funded through a program grant from Cancer Research UK. This work was supported by funding from the National Health and Medical Research Council of Australia (NHMRC) grant 390123.

\section{Author details}

${ }^{1}$ Australian Prostate Cancer Research centre-Queensland and Institute of Health and Biomedical Innovation, Queensland University of Technology, Kelvin Grove, Brisbane, Queensland, 4059, Australia. ${ }^{2}$ Molecular Cancer Epidemiology Laboratory, Queensland Institute of Medical Research, 300 Herston Road, Herston, Brisbane, Queensland, 4006, Australia.

${ }^{3}$ Gynaecological Cancer Group, Queensland Institute of Medical Research, 300 Herston Road, Herston, Brisbane, Queensland, 4006, Australia. ${ }^{4}$ Cancer Research UK Department of Oncology, University of Cambridge, Strangeways Research Laboratory, Cambridge, UK. ${ }^{5}$ Division of Cancer Epidemiology and Genetics, National Cancer Institute, Rockville, Maryland, USA. ${ }^{6}$ Department of Gynecological Oncology, University College London, Elizabeth Garrett Anderson, Institute for Women's Health, University College London, London, UK.

\section{Authors' contributions}

$J B$ organised the data, prepared the manuscript and performed sequencing and bioinformatic analyses; CMN performed statistical analyses; TOM performed the genotyping of Australian samples; MH, LMS, FL and SS helped in bioinformatic analysis and sequencing; YD and OLT performed RT PCR and sequence alignment; KB, HS, SR, SG and PP contributed to the production and analysis of the UK genome-wide association study; MAK, $A B S$ and JAC conceived the study, participated in the design and coordination of the study; ABS and JAC helped to draft the manuscript. All authors read and approved the final manuscript.

Received: 15 October 2010 Accepted: 1 April 2011

Published: 1 April 2011

\section{References}

1. Brookfield KF, Cheung MC, Koniaris LG, Sola JE, Fischer AC: A populationbased analysis of 1037 malignant ovarian tumors in the pediatric population. J Surg Res 2009, 156(1):45-49.
2. Tinelli A, Vergara D, Martignago R, Leo G, Pisano M, Malvasi A: An outlook on ovarian cancer and borderline ovarian tumors: focus on genomic and proteomic findings. Curr Genomics 2009, 10(4):240-249.

3. Lengyel E: Ovarian cancer development and metastasis. Am J Pathol 2010, 177(3):1053-1064

4. Sotiropoulou G, Pampalakis G, Diamandis EP: Functional roles of human Kallikrein-related peptidases. J Biol Chem 2009, 284(48):32989-32994.

5. Lawrence MG, Lai J, Clements JA: Kallikreins on Steroids: Structure, Function, and Hormonal Regulation of Prostate-Specific Antigen and the Extended Kallikrein Locus. Endocr Rev 2010, 31(4):407-446.

6. Clements JA: Reflections on the tissue kallikrein and kallikrein-related peptidase family - from mice to men - what have we learnt in the last two decades? Biol Chem 2008, 389(12):1447-1454.

7. Emami N, Diamandis EP: Utility of kallikrein-related peptidases (KLKs) as cancer biomarkers. Clin Chem 2008, 54(10):1600-1607.

8. Yousef GM, Scorilas A, Jung K, Ashworth LK, Diamandis EP: Molecular cloning of the human kallikrein 15 gene (KLK15). Up-regulation in prostate cancer. J Biol Chem 2001, 276(1):53-61.

9. Shaw JL, Diamandis EP: Distribution of 15 human kallikreins in tissues and biological fluids. Clin Chem 2007, 53(8):1423-1432

10. Diamandis EP, Yousef GM: Human tissue kallikrein gene family: a rich source of novel disease biomarkers. Expert Rev Mol Diagn 2001, 1(2):182-190.

11. Mavridis K, Avgeris M, Koutalellis G, Stravodimos K, Scorilas A: Expression analysis and study of the KLK15 mRNA splice variants in prostate cancer and benign prostatic hyperplasia. Cancer Science 2010, 101(3):693-699.

12. Yousef GM, Scorilas A, Katsaros D, Fracchioli S, Iskander L, Borgono C, Rigault de la Longrais IA, Puopolo M, Massobrio M, Diamandis EP. Prognostic Value of the Human Kallikrein Gene 15 Expression in Ovarian Cancer. J Clin Oncol 2003, 21(16):3119-3126.

13. Yousef GM, Scorilas A, Magklara A, Memari N, Ponzone R, Sismondi P, Biglia N, Abd Ellatif M, Diamandis EP: The androgen-regulated gene human kallikrein 15 (KLK15) is an independent and favourable prognostic marker for breast cancer. Br J Cancer 2002, 87(11):1294-1300.

14. Steven SC, Ingrid JH: A Review of Genetic Polymorphisms and Prostate Cancer Risk. Annals of epidemiology 2002, 12(3):182-196.

15. Batra J, Tan OL, O'Mara T, Zammit R, Nagle CM, Clements JA, Kedda MA Spurdle AB: Kallikrein-related peptidase 10 (KLK10) expression and single nucleotide polymorphisms in ovarian cancer survival. Int J Gynecol Cancer 2010, 20(4):529-536

16. Eeles RA, Kote-Jarai Z, Al Olama AA, Giles GG, Guy M, Severi G, Muir K, Hopper JL, Henderson BE, Haiman CA, et al: Identification of seven new prostate cancer susceptibility loci through a genome-wide association study. Nat Genet 2009, 41(10):1116-1121.

17. Lai J, Kedda MA, Hinze K, Smith RL, Yaxley J, Spurdle AB, Morris CP, Harris J, Clements JA: PSA/KLK3 AREI promoter polymorphism alters androgen receptor binding and is associated with prostate cancer susceptibility. Carcinogenesis 2007, 28(5):1032-1039.

18. Lose F, Nagle CM, O'Mara T, Batra J, Bolton KL, Song H, Ramus SJ, GentryMaharaj A, Menon U, Gayther SA, et al: Vascular endothelial growth factor gene polymorphisms and ovarian cancer survival. Gynecol Oncol 2010.

19. Song H, Ramus SJ, Kjaer SK, DiCioccio RA, Chenevix-Trench G, Pearce CL, Hogdall E, Whittemore AS, McGuire V, Hogdall C, et al: Association between invasive ovarian cancer susceptibility and 11 best candidate SNPs from breast cancer genome-wide association study. Hum Mol Genet 2009, 18(12):2297-2304.

20. TCGA Website. [http://cancergenome.nih.gov].

21. Lawrence MG, Veveris-Lowe TL, Whitbread AK, Nicol DL, Clements JA: Epithelial-mesenchymal transition in prostate cancer and the potential role of kallikrein serine proteases. Cells Tissues Organs 2007, 185(1-3):111-115.

22. Yousef GM, Diamandis EP: An overview of the kallikrein gene families in humans and other species: emerging candidate tumour markers. Clin Biochem 2003, 36(6):443-452.

23. DerSimonian R, Laird N: Meta-analysis in clinical trials. Control Clin Trials 1986, 7(3):177-188.

24. Barrett JC, Fry B, Maller J, Daly MJ: Haploview: analysis and visualization of LD and haplotype maps. Bioinformatics 2005, 21(2):263-265.

25. Kurlender L, Borgono C, Michael IP, Obiezu C, Elliott MB, Yousef GM Diamandis EP: A survey of alternative transcripts of human tissue kallikrein genes. Biochim Biophys Acta 2005, 1755(1):1-14. 
26. Porkka KP, Pfeiffer MJ, Waltering KK, Vessella RL, Tammela TL, Visakorpi T: MicroRNA expression profiling in prostate cancer. Cancer Res 2007, 67(13):6130-6135.

27. Li XF, Yan PJ, Shao ZM: Downregulation of miR-193b contributes to enhance urokinase-type plasminogen activator (UPA) expression and tumor progression and invasion in human breast cancer. Oncogene 2009, 28(44):3937-3948.

28. Rauhala HE, Jalava SE, Isotalo J, Bracken H, Lehmusvaara S, Tammela TL, Oja H, Visakorpi T: miR-193b is an epigenetically regulated putative tumor suppressor in prostate cancer. Int I Cancer 2010.

29. ESEfinder website. [http://rulai.cshl.edu/cgi-bin/tools/ESE3/esefinder.cgi? process=home].

30. TFbind webtool. [http://tfbind.hgc.jp/].

31. Olejek A, Damasiewicz-Bodzek A, Bodzek P, Wielkoszynski T, Zamlynski J, Stoltny P, Skutil M: Concentrations of antibodies against heat shock protein 27 in the sera of women with ovarian carcinoma. Int I Gynecol Cancer 2009, 19(9):1516-1520.

32. Laskowska E, Matuszewska E, Kuczynska-Wisnik D: Small Heat Shock Proteins and Protein-Misfolding Diseases. Curr Pharm Biotechnol 2010.

33. Tan OL, Whitbread AK, Clements JA, Dong Y: Kallikrein-related peptidase (KLK) family mRNA variants and protein isoforms in hormone-related cancers: do they have a function? Biol Chem 2006, 387(6):697-705.

34. Yousef GM, White NM, Kurlender L, Michael I, Memari N, Robb JD, Katsaros D, Stephan C, Jung K, Diamandis EP: The kallikrein gene 5 splice variant 2 is a new biomarker for breast and ovarian cancer. Tumour Biol 2004, 25(5-6):221-227.

35. Whitbread AK, Veveris-Lowe TL, Dong Y, Tan OL, Gardiner R, Samaratunga HM, Nicol DL, Clements JA: Expression of PSA-RP2, an alternatively spliced variant from the PSA gene, is increased in prostate cancer tissues but the protein is not secreted from prostate cancer cells. Biol Chem 2010, 391(4):461-466.

36. Rabien A, Fritzsche FR, Jung M, Tolle A, Diamandis EP, Miller K, Jung K Kristiansen G, Stephan C: KLK15 is a prognostic marker for progressionfree survival in patients with radical prostatectomy. Int I Cancer 2010.

37. Cancer Genetics Markers of Susceptibility (CGEMS) website. [http:// cgems.cancer.gov]

38. Fischer DC, Noack K, Runnebaum IB, Watermann DO, Kieback DG, Stamm S, Stickeler E: Expression of splicing factors in human ovarian cancer. Oncol Rep 2004, 11(5):1085-1090.

39. Karni R, de Stanchina E, Lowe SW, Sinha R, Mu D, Krainer AR: The gene encoding the splicing factor SF2/ASF is a proto-oncogene. Nat Struct Mol Biol 2007, 14(3):185-193.

40. Li X, Liu J, Wang Y, Zhang L, Ning L, Feng Y: Parallel underexpression of kallikrein 5 and kallikrein 7 mRNA in breast malignancies. Cancer Sci 2009, 100(4):601-607.

\section{Pre-publication history}

The pre-publication history for this paper can be accessed here: http://www.biomedcentral.com/1471-2407/11/119/prepub

\section{Submit your next manuscript to BioMed Central and take full advantage of:}

- Convenient online submission

- Thorough peer review

- No space constraints or color figure charges

- Immediate publication on acceptance

- Inclusion in PubMed, CAS, Scopus and Google Scholar

- Research which is freely available for redistribution 